\title{
Effect of Frequency on Droplet Characteristics in Ultrasonic Atomization Process
}

\author{
Amelia Sugondo ${ }^{1,2,}$, Sutrisno ${ }^{1,2}$, Willyanto Anggono ${ }^{1,2}$, and Olga Anne ${ }^{3}$ \\ ${ }^{1}$ Mechanical Engineering Department, Petra Christian University, Jl. Siwalankerto No.121-131, \\ Surabaya 60236, Indonesia \\ ${ }^{2}$ Centre for Sustainable Energy Studies, Petra Christian University, Jl. Siwalankerto No.121-131, \\ Surabaya 60236, Indonesia \\ ${ }^{3}$ Natural Sciences Faculty, Klaipeda University, Manto g. 84, Klaipeda, 92294, Lithuania, EU
}

\begin{abstract}
The study of ultrasonic atomization is one of the important factors for fuel spray process in the diesel engine combustion chamber. The droplet characteristics are influenced by liquid properties and vibration frequency. In this study, the phenomenon of droplet formation and droplet size were studied at different frequencies of the ultrasonic atomization processes for water liquid. The ultrasonic atomizer was used for the atomization process to generate droplets. CFD- 2D with the Volume of Fluid (VOF) model was used to study the process of droplet size and droplet formation at different frequencies. Water with constant film thickness and bending vibration at constant vibration amplitude were used in this model. The variation of vibration frequencies is applied from 50 $\mathrm{kHz}$ to $200 \mathrm{kHz}$ with $50 \mathrm{kHz}$ increment. The results showed that the number of droplets and the area of droplet formation increases with the increases in the vibration frequency. Effect of vibration frequency to the size of the droplet, time for droplet formation, velocity, and several droplets is more significant at vibration frequency $50 \mathrm{kHz}$ to $100 \mathrm{kHz}$ than vibration frequency from $100 \mathrm{kHz}$ to $200 \mathrm{kHz}$.
\end{abstract}

Keywords: Droplet size, formation, fuel spray, ultrasonic, vibration frequency.

\section{Introduction}

Droplets are often found in the surrounding and engineering application. In engineering application, such as pharmaceutical, biomedical, burner, and automotive, most of the droplets are generated by practical methods, such as pressure atomization, rotary atomizer, and two-fluid atomization. Those processes have some difficulty such as high energy, wider droplets size distribution, clogged, or more complex atomizer design. Recently, ultrasonic atomizers are growing faster than other atomizers because they offer more uniform and smaller droplet size. This kind of droplets characteristics is appropriated for a diesel engine. It needs more uniform droplets and small size of droplets needed to support the perfect combustion process.

There are two hypotheses in the ultrasonic atomization process, capillary wave hypothesis and cavitation hypothesis [1]. Capillary wave is formed by perpendicular

\footnotetext{
*Corresponding author: amelia@petra.ac.id
} 
vibration of a liquid thin film on the vibration surface. Boguslavskii and Eknadiosyants studied about the capillary wave hypothesis which composed of crests and troughs on the vibrating surface. However, there is not enough description of the formation of the droplets and the stage of drop detachment inside the thin film [2]. The actual droplet detachment is easy to capture only when the liquid flow rate is very low and low vibration frequency [3]. When the liquid flow rate and vibration frequency are high, it becomes difficult to capture, while the magnitude of the vibration frequency has an important role in determining the size of droplets. Yasuda observed the effect of vibration frequency and ultrasonic intensity to the atomization rate and the number of atomized droplets. They concluded that vibration frequency affected more than ultrasonic intensity [4]. However, due to the lack of studies conducted, empirical correlations are mainly used to predict droplet size and droplet distribution. This causes difficulty to precisely predict the size and distribution of droplet $[5,6]$.

The volume of Fluid (VOF) is one of the models available to model a multiphase fluid using Computational Fluid Dynamic (CFD). It can be used to predict the shape and motion of the droplets on the steady or transient process. VOF can also be used to track free fluid surface [7]. To get accurate tracking method and to capture the shape precisely should apply smaller meshing size $[3,8,9]$.

\section{Numerical simulation for ultrasonic atomization spray}

The results of ultrasonic atomization process can be solved in three ways, such as analytical, experimental, and numerical approach. The numerical approach is used to predict the phenomena of droplet formation since the disintegration of the ultrasonic atomization process is not clear yet. The approach uses Computational Fluid Dynamic (CFD), it is useful to solve many engineering problems which cannot be solved or difficult to use experimental and analytical approach or to verify those approach.

\subsection{Volume of the fluid model}

The volume of Fluid model (VOF) is a multiphase model that is categorized as a very robust model, simple and powerful methods [10]. The advantage of applying VOF does not require additional equation $[6,7,10]$; it uses a single momentum equation (1). The equation is depended on the volume fraction of all phases through the density $\rho$ and viscosity $\mu$. VOF can be tracked as a free fluid surface and cover moving boundary model [7]. The vibration for generating droplet of ultrasonic atomization process can be utilized by using dynamic meshing to move the boundary. The moving boundary is assigned by using Equation (2).

$$
\frac{\partial}{\partial t}(\rho \vec{v})+\nabla \cdot(\rho \vec{v} \vec{v})=-\nabla p+\nabla \cdot\left[\mu\left(\nabla \vec{v}+\nabla \vec{v}^{T}\right)\right]+\rho \vec{g}+\vec{F}
$$

The requirement for capturing droplet accurately and precisely can be done with fine meshing size that is around one-fifth from diameter droplets of the size of the droplets with uniform spaced mesh [3, 9]. VOF is available for pressure based analysis. Equation (2) is the continuity equation for the volume fraction of the phases to track the interfaces between the phases. 


$$
\frac{1}{\rho_{y}}\left[\frac{\partial}{\partial t}\left(\alpha_{y} \rho_{y}\right)+\nabla \cdot\left(\alpha_{y} \rho_{y} \overrightarrow{\mathrm{v}}_{y}\right)=S_{\alpha y}+\sum_{x=1}^{n}\left(\dot{m}_{x y}-\dot{m}_{y x}\right)\right]
$$

Where $\dot{m}_{y x}$ is the mass transfer from phase y to phase $\mathrm{x}$ and $\dot{m}_{x y}$ is conversely. $S_{\alpha \mathrm{y}}$ is zero or constant of user-defined mass source for each phase. Surface tension is set to capture the capillary phenomena. For small dimension is needed to activate double precision version in option simulation.

For standard finite-difference interpolation schemes in an explicit approach, the volume fraction values can be applied by using Equation (3)

$$
\frac{\alpha_{y}^{n+1} \rho_{y}^{n+1}-\alpha_{y}^{n} \rho_{y}^{n}}{\Delta t}+\sum_{f}\left(\rho_{y} U_{f}^{n} \alpha_{y, f}^{n}\right)=\left[S_{\alpha y}+\sum_{x=1}^{n}\left(\dot{m}_{x y}-\dot{m}_{y x}\right)\right] V
$$

Where $n+1$ is an index for current time step, $n$ is an index for previous time step, $\alpha_{y, f}$ is the face value of the $y^{\text {th }}$ volume fraction, $V$ is the volume of the cell, and $U_{f}$ is volume flux through the face.

\subsection{Model for ultrasonic atomization spray}

Model of this research is built only for the ultrasonic atomization surface, which is vibrated at specified frequencies. The model domain is built in for rectangular shape of 2 Dimensional (2D) by using Gambit software. The length and width of the model are $0.004 \mathrm{~m}$ and $0.0004 \mathrm{~m}$, respectively. The boundaries of the model are walls for the vertical and the bottom sides and pressure outlet for the top side. Figure 1 shows the detail of the model. The bottom surface is vibrated at an ultrasonic frequency, and the surface is covered by $0.00004 \mathrm{~m}$ of the liquid film thickness.

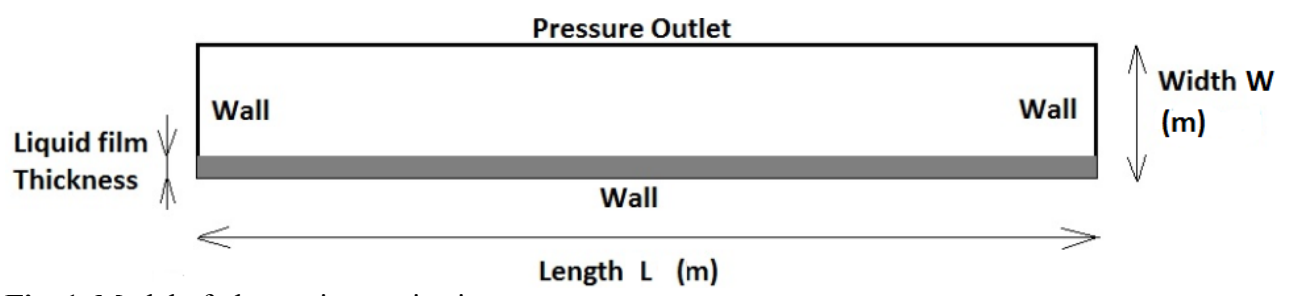

Fig. 1. Model of ultrasonic atomization spray

The quadrilateral meshing type is chosen for this model. The mesh type is chosen by considering numerical diffusion, computational expense, set up time, and the geometry. The quadrilateral mesh is chosen for this model since the model is applied for the top surface of the model. The number of element meshing is 400000 elements. Water is used for the atomization fluid. The properties of water are $998.2 \mathrm{~kg} \mathrm{~m}^{-3}$ for density, $0.001003 \mathrm{~kg} \mathrm{~m}^{-1} \mathrm{~s}^{-1}$ for the viscosity and $0.0728 \mathrm{~N} \mathrm{~m}^{-1}$ for surface tension.

\subsection{Dynamic meshing}

Boundary motion can be achieved by using dynamic meshing. User Defined Function is used to deform a rigid body. Refer to the equation $y=A \cdot \sin (2 \pi f t)$ where $A=A^{\prime} \cdot\left(-x^{2}+L x\right)$ is amplitude, $A^{\prime}$ is adjusted amplitude, $f$ is frequency and $t$ is time [10], the function is written in c language. That equation should be compiled when it is applied VOF model in 
CFD. The setting parameters of vibration frequency are from $50 \mathrm{kHz}$ to $200 \mathrm{kHz}$ with $50 \mathrm{kHz}$ increment at constant vibration amplitude.

\section{Results and discussion}

Figure 2 shows the simulation results of liquid disintegration phenomena. The surface of the liquid film generates wave at the surface that is called capillary surface waves. The capillary waves are generated when the liquid surface is disturbed. The energy to disturb should be higher than the liquid surface tension. From Figure 2, it can be seen that the number of capillary waves for low vibration frequency is less than that of high vibration frequencies.

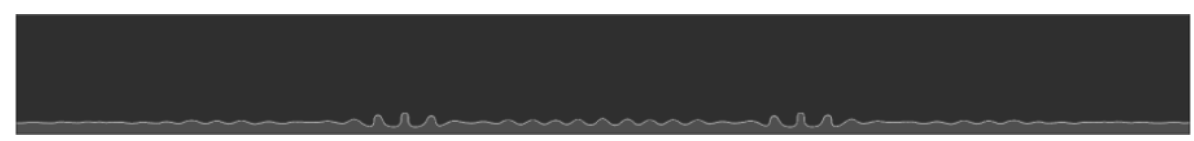

a. frequency $50 \mathrm{kHz}$

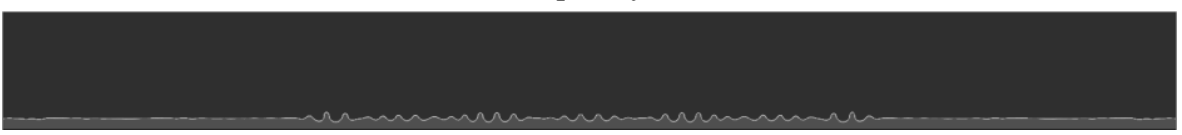

b. frequency $100 \mathrm{kHz}$

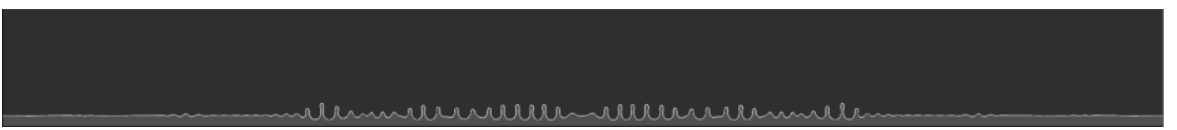

c. frequency $150 \mathrm{kHz}$

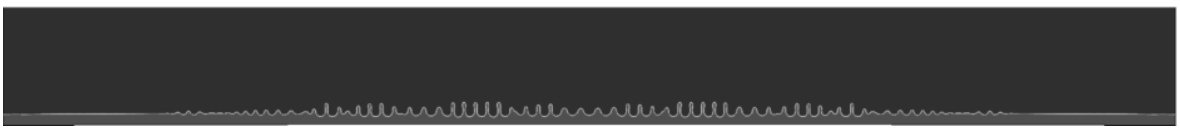

d. frequency $200 \mathrm{kHz}$

Fig. 2. Comparison of generation capillary wave of different frequencies.

Figure 3 shows the effect of vibration frequency to the velocity from the simulation results. The trend of the velocity with the function of frequency shows a linear correlation between these parameters. Vibration energy is converted into velocity, which will affect the kinetic energy. That refers to equation EK $=0.5 \mathrm{~V}^{2}=0.5(2 \pi \mathrm{fA})^{2}$ where EK is kinetic energy, $\mathrm{V}$ is velocity, $\mathrm{f}$ is vibration frequency, and $\mathrm{A}$ is vibration amplitude. In the ultrasonic atomization, the increase of velocity will be followed by a drop of static pressure, which helps the evaporation process. The effect of liquid surface tension must be greater than the pressure drops to avoid cavitation. When the pressure drop is more dominant than the evaporation process, and consequently, the droplet size is larger and irregular. $\mathrm{m} \mathrm{s}^{-1}$ 


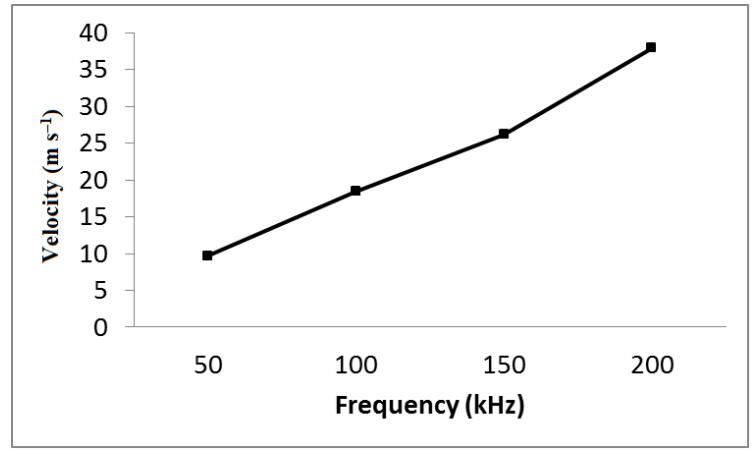

Fig. 3. Effect of the vibration frequency to the velocity.

The number of droplet particles is influenced by a number of capillary waves. Figure 4 shows that most of the crests of the capillary wave generate droplets when the energy is enough to overcome the surface tension of the liquid. The crest of capillary wave oscillates to form crest and trough alternately, as shown in the dashed line series in Figure 4. When the energy is sufficient to stretch the crest, each crest changes to form a ligament. The ligaments are increasingly attracted to form a thin neck and eventually rupture into the droplet.

a.

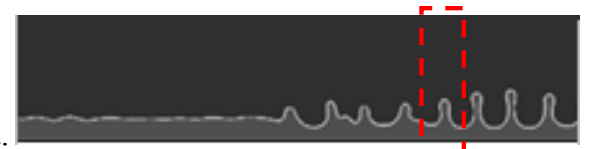

b.

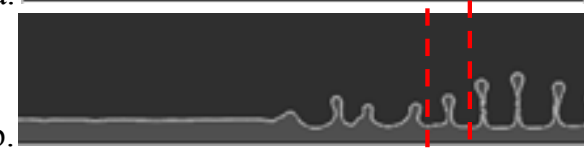

c.

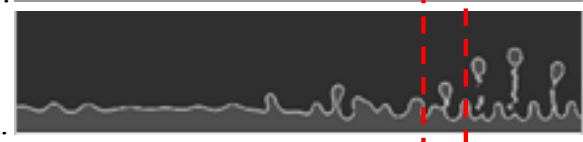

d.

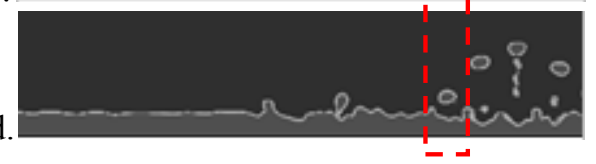

Fig. 4. Process of droplet generation at vibration frequency $100 \mathrm{kHz}$, a. capillary waves, b. ligaments, c. rupture droplets, d. droplets

Effect of the frequency to the number and the size of droplets can be seen in Figure 5. At high vibration frequency is generated more droplets than at low vibration frequency. Vibration frequency affects energy to generate capillary waves. The number of capillary waves increases when the wavelength becomes shorter. The equation $\lambda=\mathrm{c} / \mathrm{f}$ is used to prove the relationship between vibration frequency $f$ and wavelength $\lambda$ in the phenomenon of ultrasonic atomization. The consequence of reducing capillary wavelength will reduce the size of the droplets because the size of each crest becomes smaller. Fig. 5 shows a significant drop in the size of droplet around $40 \%$ for vibration frequency $50 \mathrm{kHz}$ to $100 \mathrm{kHz}$, however when the vibration frequency increases from $100 \mathrm{kHz}$ to $150 \mathrm{kHz}$ and $150 \mathrm{kHz}$ to $200 \mathrm{kHz}$, the size of droplet decreases by about $20 \%$ and $5 \%$, respectively. The 
number of droplets has increased significantly with increasing vibration frequency, as shown in Figure 5.

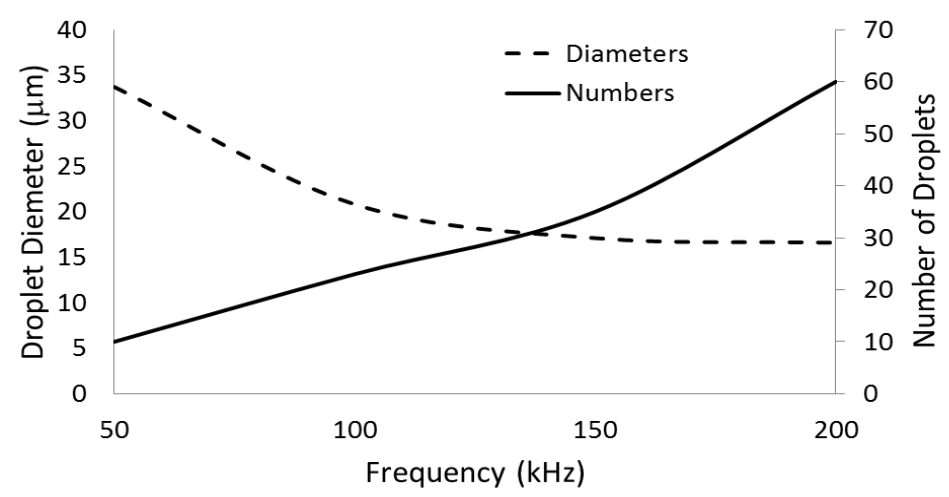

Fig. 5. Effect of vibration frequency to the number of droplets

Based on the time to generate capillary waves, droplets formation process at low vibration frequency takes longer than at high frequency (Figure 6). Energy conversion from the vibration frequency must be higher than water surface tension to disrupt the water surface. In this study, the time to generate droplet takes at least $19500 \mu$ s for vibration frequency $50 \mathrm{kHz}$, whereas it took only $3000 \mu$ s for vibration frequency $200 \mathrm{kHz}$. It is predicted that the vibration energy of the capillary wave was not able to rupture the liquid surface tension bond for low vibration frequency. Vibration energy should overcome the surface tension forces to form capillary wave until it ruptures into droplets.

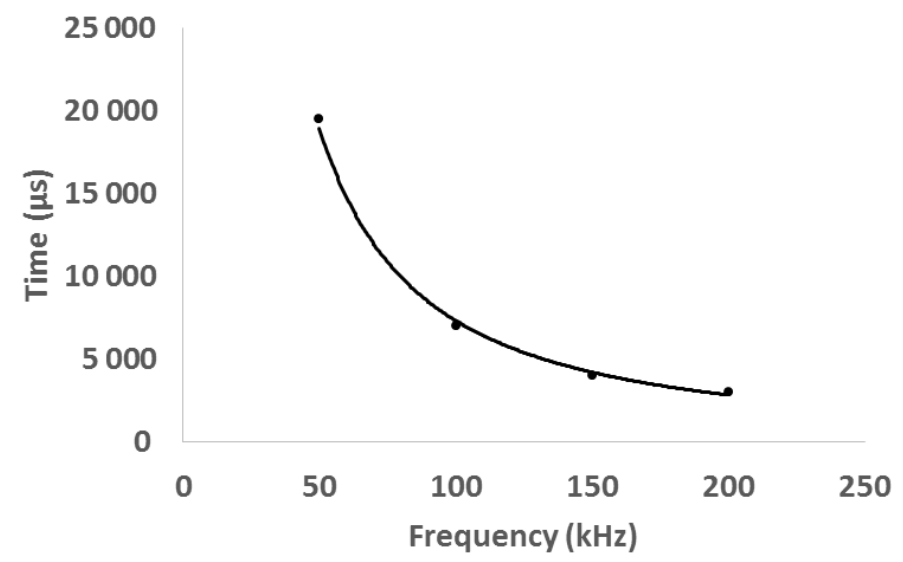

Fig. 6. The time difference for several vibration frequencies to form the droplets

\section{Conclusions}

Effect of increasing vibration frequencies from $50 \mathrm{kHz}$ to $100 \mathrm{kHz}$ has more impact on the velocity, size of the droplet, and time for droplet formation than increasing vibration 
frequencies from $100 \mathrm{kHz}$ to $200 \mathrm{kHz}$. The increase of velocity up to two times at vibration frequency less than $100 \mathrm{kHz}$, while the size of the droplets decreases up to $40 \%$. The impacts to droplets size decrease slowly when the vibration frequency increase from 100 $\mathrm{kHz}$ to $200 \mathrm{kHz}$. The number of droplets increases significantly with increasing vibration frequencies. It almost shows a linear correlation. While the time for droplet formation requires $19500 \mu \mathrm{s}$ at $50 \mathrm{kHz}$ and only $3000 \mu$ s at $200 \mathrm{kHz}$ of vibration frequencies.

\section{References}

1. K.A. Ramisetty, A.B. Pandit, P.R. Gogate. Ultrasonic Sonochemistry, 20:254264(2013). https://www.sciencedirect.com/science/article/pii/S1350417712000909

2. D.B. Gutierrez, V.S. Barrera, Y.V. Hernandez, L.G. Garreton, C.Z. Iglesias, Physics Procedia 63:37-41(2015). https://www.sciencedirect.com/science/article/pii/S1875389215000772

3. J. Shinjo, A. Umemura. International Journal of Multiphase Flow, 36,7:513532(2010). https://www.sciencedirect.com/science/article/pii/S0301932210000637

4. K. Yasuda, H. Honma, Z. Xu, Y. Asakura, S. Koda. Japanese J. Appl. Phys., 50,07HE23:1-5(2011) https://iopscience.iop.org/article/10.1143/JJAP.50.07HE23/meta

5. A.H. Lefebvre, V.G. McDonell. Atomization and Spray. USA: CRC Press (2017) p.1-16. https://www.taylorfrancis.com/books/9781498736268

6. M. Tembley, C. Lecot, A. Soucemarianadin. Applied Thermal Engineering, 31,5: 656-667(2011).

https://www.sciencedirect.com/science/article/pii/S1359431110004230

7. N. Ashfriz. Numerical techniques for simulating the atomization process. In:

Handbook of atomization and sprays. N. Ashfriz (Ed). Heidelberg, Germany: Springer Science \& Business Median (2011). p. 339-357. https://www.springer.com/us/book/9781441972637

8. B.A. Nichita, I Zun, J.R. Thome. A VOF Method Coupled with a Dynamic Contact Angle Model for Simulation of Two-Phase Flows with Partial Wetting, $7^{\text {th }}$ International Conference of Multiphase Flow. (Tampa, Florida, USA, 2010). https://infoscience.epfl.ch/record/150243/files/NichitaZT-ICMF2010.pdf

9. A. Sugondo, M. Nakajima, T. Yung, M. Leorna. A comparison droplet formation for flexural curvature vibration and flat surface vibration by numerical simulation. $13^{\text {th }}$ Triennial International Conference of Liquid Atomization and Spray System (Tainan, Taiwan, 2015). p. 1-8. http://ilasseurope.org/events/13th-iclass-2015/

10. V. Srinivasan, A.J. Salzar, K. Saito. Applied Mathematical Modelling, 35,8:3710-3730 (2011) https://www.sciencedirect.com/science/article/pii/S0307904X11000540 